\title{
ILCEA
}

Revue de l'Institut des langues et cultures

d'Europe, Amérique, Afrique, Asie et Australie

$12 \mid 2010$

La FASP : dix ans après...

\section{La validation du substrat professionnel dans $L a$ proie de Michael Crichton (Prey, US, 2002)}

\section{Stéphanie Genty}

\section{OpenEdition \\ Journals}

Édition électronique

URL : http://journals.openedition.org/ilcea/489

DOI : 10.4000/ilcea.489

ISSN : 2101-0609

Éditeur

UGA Éditions/Université Grenoble Alpes

Édition imprimée

ISBN : 978-2-84310-180-9

ISSN : 1639-6073

Référence électronique

Stéphanie Genty, "La validation du substrat professionnel dans La proie de Michael Crichton (Prey, US, 2002) », ILCEA [En ligne], 12 | 2010, mis en ligne le 29 septembre 2010, consulté le 24 avril 2019. URL http://journals.openedition.org/ilcea/489 ; DOI : 10.4000/ilcea.489

Ce document a été généré automatiquement le 24 avril 2019

(C) ILCEA 


\title{
La validation du substrat professionnel dans La proie de Michael Crichton (Prey, US, 2002)
}

\author{
Stéphanie Genty
}

\begin{abstract}
«Tout comme la guerre est trop importante pour être laissée aux simples généraux, la science est trop importante pour être laissée aux simples scientifiques ${ }^{2} »$
\end{abstract}

\section{Introduction}

1 En 1999, Michel Petit a identifié comme de la Fiction à substrat professionnel ou FASP tout thriller contenant des éléments extra- et intra-textuels relevant de la représentation authentique d'un milieu professionnel donné. Le lecteur est censé y trouver un portrait réaliste d'un univers pour lequel l'auteur a été formé ou dont il est issu. L'auteur est reconnu par des experts, par ses pairs, et souvent par les médias comme étant un spécialiste au même titre que les professionnels non-créateurs de fiction. Il peut même être considéré comme étant plus crédible que ces experts dans la mesure où il ne fait plus tout à fait partie du milieu et peut donc porter un regard informé mais critique sur la profession ${ }^{3}$. L'authenticité du substrat garantit son succès. En effet, dans «La fiction à substrat professionnel: une autre voie d'accès à l'anglais de spécialité ", Michel Petit souligne la manière dont l'authenticité de ce substrat peut être mise en avant dans la démarche commerciale des maisons d'édition (Petit, 1999, p. 65).

2 En tant qu'objet d'étude, la FASP nous fournit une quantité d'informations sur un milieu professionnel et sur ses acteurs, informations qui nous permettent de mieux cerner et la langue et la culture de ce milieu. D'un point de vue pédagogique, la FASP constitue pour nos étudiants un regard sur leur univers post-universitaire. Et c'est justement en vue d'une utilisation pédagogique que la question de la validation doit être soulevée. Dans l'article «Quelques réflexions sur la fiction à substrat professionnel: du général au 
particulier ", Michel Petit écrit que cette fiction est " produite par le milieu lui-même à travers l'action de certains de ses membres devenus créateurs de fiction » et ajoute qu'il faut néanmoins « déterminer dans quelle mesure le message est celui de la profession » et dans quelle mesure l'auteur-créateur peut être pris comme porte-parole de celle-ci (Petit, 2004, p. 22). Pour ce faire, nous devons évaluer la place de l'auteur dans la profession et la façon dont ses œuvres sont perçues par ses pairs. C'est ce que nous nous sommes donné comme objectif dans cet article, et pourquoi nous avons décidé de faire appel aux spécialistes.

\section{Michael Crichton et La proie}

3 Michael Crichton (1948-2008) fait partie des auteurs de FASP qui ont décidé très tôt d'arrêter une carrière scientifique pour se consacrer à l'écriture. Diplômé de Harvard en 1964, il a passé un an à Cambridge en tant que « Visiting Lecturer » en anthropologie avant de poursuivre ses études au Harvard Medical School où il a été diplômé en 1969. Il a ensuite effectué une année post-doctorale au Salk Institute for Biological Studies, participant à des recherches sur la politique publique. La biographie affichée sur le site web de l'auteur indique qu'il a fait une étude numérique d'un crane égyptien à Harvard dans les années soixante et qu'il a publié dans Metabolism. L'écrivain maîtrisait donc les techniques de rédaction scientifique médicale, techniques qu'il remet en question dans un article paru dans le New England Journal of Medecine sur l'obscurité dans la communication médicale («medical obfuscation ») et où il note: "La rédaction médicale est une tentative calculée et très complexe pour embrouiller le lecteur "; "l'obscurité médicale servirait d'outil de reconnaissance pour les membres d'un groupe, un peu comme une poignée de main pour quelque société secrète ${ }^{4} »$. Le fait que Crichton s'interroge sur la nature de la communication scientifique, et notamment sur l'opacité qui lui est associée et qui limite son accès à une communauté d'initiés, ne peut qu'intriguer. Convaincu que la science et la technologie étaient au cœur des changements sociaux et qu'il fallait comprendre leurs enjeux pour prendre des décisions politiques adéquates, le romancier était préoccupé par l'accès du grand public à des informations scientifiques souvent complexes. En tant qu'écrivain, il s'est donné comme mission d'informer ses lecteurs des dangers potentiels des évolutions scientifiques.Il en dit autant dans un entretien avec Charlie Rose à propos de La proie (26 novembre 2002) 5 .

Le rapport complexe qu'entretient cet auteur avec le réel scientifique a évolué avec chaque publication. Dans La souche d'Andromède (1969), une contamination de la Terre par des microbes venus de l'espace est présentée comme un fait tenu secret par les autorités, avec références inventées à l'appui, un cas de «faux documents ». Plus récemment, dans État d'urgence (2004), des études scientifiques véritables, mais partielles (et donc partiales), servent à étayer un scénario catastrophe écologique très polémique. Des climatologues se sont insurgés contre le détournement d'une réalité scientifique dans le roman. Dans La proie (2002), l'auteur choisit de traiter les nanotechnologies, autre source d'angoisse pour le public américain, surtout quand elles se mêlent à la biologie et à l'intelligence artificielle. Crichton met en scène la perte de contrôle par les inventeurs d'un essaim de nanoparticules carnivores capables de se reproduire tout en évoluant par rapport à leur environnement. Ces particules sont programmées pour apprendre et, en tant que prédateurs, trouvent mille stratégies pour s'emparer de nous, leur proie. 
L'essaim parasitique se loge ainsi à l'intérieur du corps humain afin de prendre le contrôle de celui-ci et d'étendre son pouvoir, dans une dangereuse forme de « symbiose ».

Comme d'autres romans du même auteur, La proie comprend un "cadre savant » qui étaye la crédibilité de la fiction et donc le message de l'auteur. D'emblée, des citations d'experts et une introduction sur l'évolution artificielle au xxI siècle (par Crichton) nous mettent en garde contre les dangers potentiels des nano- et biotechnologies. Des notes de bas de page soutiennent les propos de Crichton et des références bibliographiques suivent le récit pour tout lecteur qui aimerait en savoir plus. Ces références en langue anglaise ne sont pas incluses dans la version française du roman, à la différence d'État d'urgence dont la traduction comprenait l'important appareil bibliographique de la version anglaise pour les lecteurs intéressés. L'inclusion de telles références sert à renforcer la crédibilité de l'auteur et de son message. Elles constituent la trace tangible de ses recherches et la preuve qu'il parle au nom d'une communauté. Le cadre de La proie laisse entendre que Crichon ne serait pas le seul à parler des dangers potentiels des nanotechnologies : Eric Drexler, un des pères fondateurs de la nanoscience, ainsi que J. Doyne Farmer et Aletta d'A. Belin, theoriciens de la vie artificielle, l'avaient fait avant lui (Drexler, 1986 ; Farmer et Belin, 1992). Un autre penseur, qui ne figure ni dans les notes ni dans la bibliographie de Crichton avance les mêmes craintes: Bill Joy, inspiré par une conversation avec Ray Kurzweil ${ }^{6}$, et qui a publié un article intitulé «Why the Future Doesn't Need Us» (Joy, 2000). Entouré de ces spécialistes, Crichton semble autorisé/s'autoriser à spéculer sur les dérives d'une science nouvelle.

\section{Objectifs et démarche}

6 Nous avons voulu mettre La proie à l'épreuve en soumettant l'ouvrage à la lecture attentive de collègues experts dans les différents domaines décrits par Crichton dans son roman. Nous avons voulu évaluer dans quelle mesure Crichton réussit à représenter la réalité de milieux professionnels scientifiques différents, en l'occurrence non-médicaux, c'est-à-dire en dehors des compétences de l'auteur. Notre objectif était de voir dans quelle mesure l'auteur réussit à représenter ces milieux scientifiques afin d'explorer la notion d'hybridisation et ceci à plusieurs niveaux : l'intrigue, les personnages ainsi que leur comportement et leurs modes de communication, les lieux et le contenu scientifique. Enfin, nous avons voulu interroger des collègues sur l'utilisation de cette fiction en salle de cours avec des étudiants qui se destinent à la recherche.

7 L'accès aux spécialistes a été aisé en raison de la présence dans notre établissement d'unités de recherche telles que le Laboratoire de l'étude des milieux nanométriques (LMN). Un questionnaire a été élaboré et distribué à plusieurs collègues de l'université ainsi qu'à des amis travaillant dans les domaines évoqués par Crichton: en nanotechnologie, en biologie et en informatique. Nous leur avons demandé de lire le roman et de répondre au questionnaire. Nous nous sommes vus ensuite pour en discuter. Les scientifiques qui ont participé à cette étude sont: Philippe Houdy, Professeur physicien, spécialiste des nanosciences, ancien directeur du Laboratoire de l'étude des milieux nanométriques (LMN) à l'université et auteur de plusieurs livres sur le sujet ${ }^{7}$; Pascal Aubert (MCF-HDR), également universitaire, directeur actuel du Laboratoire LMN ; Serge Jard, Professeur de Biologie à la retraite depuis 1997 (CNRS-INSERM-Université de Montpellier 1 et 2) ayant travaillé sur la pharmacologie moléculaire; Claude Jard, Ingénieur en télécommunications, Directeur de recherche CNRS en informatique et 
mathématiques, professeur à l'ENS Cachan (antenne de Bretagne) ayant travaillé sur les systèmes répartis et les réseaux. Ce dernier était le seul à avoir déjà lu le roman. Les autres n'avaient jamais rien lu de Michael Crichton.

8 Avant les entretiens, nous avons procédé à la validation des éléments qui entourent le récit de Crichton: les citations qui ouvrent le roman, les notes et références bibliographiques, l'existence des scientifiques mentionnés et l'état des recherches menées dans les différents domaines. Nos recherches nous ont permis de confirmer la véracité de ces éléments. De plus, nos lectures de l'article de Bill Joy et des informations sur Eric Drexler nous ont convaincue que d'autres que Crichton s'inquiètent des évolutions possibles des technologies moléculaires associées à la biologie et aux avancées en informatique.

\section{Résultats}

9 Si l'auteur se base sur de vraies recherches et s'appuie sur des avertissements émis par des scientifiques, reste à savoir si le roman lui-même est convaincant. Dans ce cas précis, les experts que nous avons interrogés sont unanimes pour dire que l'intrigue imaginée est impossible. Si elle se base sur des programmes de recherche réels et pose de vraies questions - parfois les mêmes que celles qui se posent les scientifiques - l'auteur passe néanmoins très vite à la fiction. Ainsi certains éléments relevant de la nanoscience ou de l'intelligence artificielle sont vrais, mais l'assemblage de ces éléments ne marche pas. Pour Pascal Aubert, trois aspects de l'intrigue sont discutables: l'existence des nano robots, leur comportement collectif et leur symbiose avec le corps humain. Philippe Houdy dénonce ce qu'il qualifie de "spéculation abusive »: «l'auteur met en scène la toute puissance néfaste de robots intégrant sans limite ni contrainte, donc sans aucun réalisme, toutes les potentialités du monde $\mathrm{NBIC}^{8}$.» L'intrigue, fondée sur une "fuite » intentionnelle de nanoparticules dans la nature, où elles se reproduisent, est jugée impossible à la fois en raison des procédures suivies par tout scientifique sérieux et en raison des contrôles. Les nanosciences étant très réglementées, il serait impossible de décider de "lâcher" un essaim de nanoparticules dans la nature pour observer son évolution. De plus, selon nos collègues, si des nanoparticules étaient accidentellement libérées dans la nature, elles se dissiperaient dans l'air et seraient inoffensives. Selon eux, ce risque est anticipé par les scientifiques, prévu et contrôlé, depuis les débuts des nanosciences, un avis qui n'est cependant pas partagé par tous'. Ceci étant, les lecteurs spécialistes sont d'accord pour dire que malgré son manque de réalisme, l'intrigue est néanmoins très réussie, car l'auteur sait tenir son auditoire en haleine. Seulement, l'imaginaire domine, et la fiction prend largement le dessus sur la science.

La représentation du laboratoire et des personnages scientifiques s'est révélée tout aussi problématique. L'isolement du centre d'assemblage moléculaire dans le désert pose problème car une telle configuration est jugée impossible en Europe. Un laboratoire de nanotechnologie, très complexe, implique des démarches administratives importantes, des autorisations et des contrôles à plusieurs niveaux. De plus, l'équipe fictionnelle de chercheurs n'est crédible ni par son nombre, ni par sa composition, ni par les compétences qu'elle réunit. Les huit personnes qui la constituent ne comptent pas de spécialistes en nanosciences, mais une biologiste spécialiste des primates, un ingénieur et une poignée d'informaticiens aux compétences variées. Ils ne sont pas habillés de façon adéquate pour la recherche en nanotechnologie et ne réagissent pas en scientifiques 
responsables. L'équipe est menée par un industriel, Ricky Morse, et les enjeux sont à la fois personnels, militaires et commerciaux. Dans la réalité, un tel laboratoire emploierait entre cent et mille personnes; elles seraient habillées en "cosmonautes» et travailleraient dans de petits espaces clos séparés par des sas, jamais dans une grande salle. Pour Serge Jard, seule Mae Chaeng, absorbée par sa recherche et par le devenir de l'essaim est crédible. Selon nos interlocuteurs, les personnages de Crichton relèvent du fantasme populaire. L'auteur dit avoir voulu réactualiser le conte de Frankenstein dans la création de ses monstres modernes. Pour nos collègues, ce sont soit des «images d'Epinal », soit des "savants fous». Seul Pascal Aubert n'était pas choqué par la représentation fictionnelle de ses collègues américains.

11 Cependant, le modèle de Los Alamos dans le Nouveau Mexique aurait pu servir de modèle à Crichton pour justifier l'isolement du laboratoire et son caractère confidentiel. Dans La proie, la société Xymos travaille sur un contrat qui est militaire à la base mais qui a des applications médicales et commerciales. Dans un contexte nord-américain des fonds conséquents pourraient être réunis à titre privé ou public pour une recherche tenue secrète selon le modèle de Los Alamos, ce qui validerait le choix du lieu. Toutefois, le taux de financement évoqué par Crichton relève de la pure fiction: ses protagonistes réunissent auprès d'investisseurs privés onze milliards de dollars. À titre comparatif, à Grenoble, le laboratoire Minatec - qui est le premier laboratoire européen en nanotechnologie et le $3^{\mathrm{e}}$ mondial - a investi 150 millions d'euros en 3 ans (2002-5) et récemment a récolté 46,5 millions dans le cadre du projet Nano Solar Crystal ${ }^{10}$ ainsi que 2,3 milliards d'euros pour le programme « Nano 2012 » (2008-2012), 340 millions d'euros de STMicroelectronics et 457 millions d'euros de l'État et des collectivités territoriales ${ }^{11}$.

qui concerne le contenu scientifique, le récit mélange du vrai et du faux. Pour Philippe Houdy, «l'auteur retient ce qui convient à sa thèse et rejette tout ce qui ne l'arrange pas et ceci sans aucune rigueur scientifique». Nous pouvons énumérer trois failles importantes dans la construction de l'intrigue: 1) Bien que les nanoparticules existent, un nuage ou essaim de celles-ci ne pourrait pas exister en raison d'un problème de stabilité (il a fallu 4 milliards d'années pour que les molécules se stabilisent) ;2) Il est impossible que ces nanoparticules se ressourcent à partir d'énergie solaire en raison de leur taille (ceci serait possible sur une échelle millimétrique, mais non sur une échelle nanométrique et 3) Il existe des problèmes liés au phénomène de l'intelligence distribuée. On est tenté d'établir une analogie entre le monde animal et le monde technologique : on imagine qu'à partir d'un certain nombre d'entités ces dernières s'organiseront entre elles pour former un ensemble capable de " penser » et de "se reproduire ». Les cellules et les molécules "communiquent» entre elles en s'envoyant des signaux, comme le font les virus informatiques nous rappelle Crichton. Ceci est vrai, mais n'est pas possible avec des nano molécules.

13 Enfin, le récit est affaibli par un grand nombre d'inexactitudes que le lecteur nonspécialiste aura du mal à repérer. Par exemple, deux types de microscope sont confondus : l'auteur évoque tantôt un STM ou « scanning tunneling microscope ", tantôt un SEM « scanning electron microscope ». Or il s'agit de deux technologies différentes et complémentaires. De même pour deux formes d'énergie solaire: il confond les technologies piézoélectrique et photovoltaïque. Sur le plan biologique, un domaine pourtant connu de Crichton, il affirme qu'une réaction cutanée intense ne provoque pas de mobilisation d'anticorps ou de globules blancs, ce qui est faux. Si un bébé souffrait d'une allergie telle que celle décrite dans le roman pour le bébé Amanda, il y aurait 
forcément une mobilisation des défenses immunitaires. La réaction cutanée en est d'ailleurs une manifestation. L'auteur dit constater que les insectes ne sont pas génétiquement programmés pour se comporter d'une certaine façon, pour construire des fourmilières, par exemple, mais ils le sont. En matière de physique, les spécialistes ont soulevé plusieurs problèmes : il est très difficile pour des atomes de traverser un liquide ; il est impossible que des composants à l'intérieur d'un ordinateur soient corrodés comme le décrit le roman ; il n'est pas sûr que l'on puisse pulvériser des nanoparticules avec un liquide contenant des isotopes ; la pression atmosphérique décrite par Crichton est tantôt mal exprimée (il ne donne pas la surface), tantôt trop basse (équivalent à une salle normale). Enfin, certains éléments scientifiques pourraient constituer des «effets de réel » ou des « effets FASP ${ }^{12}$ ». À titre d'exemple, un champ magnétique n'a aucune raison scientifique d'exister dans ce laboratoire : un tel champ ne sert à rien. L'architecture est " fractale ", mais on ne sait pas pourquoi. Et on n'a pas besoin de températures très basses pour la fabrication de nano robots. Même en ce qui concerne la langue, Crichton « utilise des mots et des concepts inadéquats pour paraître scientifique ", selon Houdy. Pour Serge Jard, ce n'est pas de la langue de spécialité : tout est compréhensible pour le nonspécialiste.

Tous ceux que nous avons interrogés nous mettent en garde contre une utilisation pédagogique du roman, sauf en tant que "contre-modèle ». Leur réticence n'est pas uniquement due aux manquements scientifiques. Il y a aussi des considérations psychologiques, éthiques et philosophiques. Ils sont d'accord sur le fait que Crichton exploite nos peurs des nanosciences, celle des robots et celle de perdre tout contrôle sur nos innovations technologiques. Mais Houdy va plus loin. Pour lui, « l'auteur est excellent pour faire vibrer les pires angoisses de l'homme", parmi celles-ci, la peur du morcellement, de la possession par un autre et la perte de contrôle psychique. De ce fait, "l'auteur, avec son discours pseudo-scientifique est donc dangereux si les lecteurs le prennent au sérieux ». Ce collègue se méfie beaucoup de la manipulation par l'auteur de nos émotions.

Pascal Aubert soupçonne Crichton d'héberger Dieu derrière son histoire de Frankenstein contemporain. Le fait que les nanoparticules soient créées puis laissées libres d'évoluer dans leur environnement semble indiquer une croyance en un "dessein intelligent" ( intelligent design»). Crichton le dit assez clairement dans l'entretien consacré au roman qu'il a accordé à l'émission de Charlie Rose. Il s'intéresse à la façon dont un « minimum » d'éléments laissés entre eux se développent sans l'intervention de l'homme. Cette idée a servi de base pour le scénario du roman. Dans le récit, c'est l'algorithme génétique du logiciel FEDPREY qui explique l'évolution des essaims de nanoparticules.

\section{Discussion}

Compte tenu des dysfonctionnements au sein de l'intrigue et des nombreuses erreurs scientifiques, il s'avère que, dans le cas précis de La proie, Michael Crichton ne s'est pas suffisamment entretenu avec les spécialistes. Non seulement son récit comprend des inexactitudes en matière de physique, mais il en comprend aussi dans le domaine d'expertise de l'auteur, à savoir la médecine. Notre biologiste ne reconnait pas Michael Crichton comme faisant partie de sa communauté scientifique, et encore moins nos physiciens et notre informaticien. L'auteur comprend suffisamment les recherches dans ces différentes disciplines pour en fabriquer une fiction, mais leur substrat professionnel 
est faible. Nous avons pu vérifier le contenu du cadre du récit et pouvons confirmer que Michael Crichton constitue une voix parmi d'autres qui prônent davantage de prudence et de contrôles en matière de recherche en nano- et biotechnologies. Mais l'on ne peut pas dire que Crichton constitue un porte-parole ou que son message soit celui de «la profession ».

17 Si l'intrigue du roman s'inspire des recherches actuelles dans les différents domaines décrits, d'autres préoccupations prennent le dessus. Elles peuvent être commerciales, politiques ou narratives. Ainsi, on peut s'interroger sur la qualité du travail de l'éditeur ou, tout au moins, sur les choix éditoriaux : pour quelles raisons a-t-on négligé la rigueur scientifique? Y avait-il des contraintes de temps liées à une date de publication? L'éditeur a-t-il voulu profiter de "l'effet 11 septembre » en sortant son roman un an après l'événement? C'est l'avis d'un de nos spécialistes. L'éditeur jugeait-il cette fiction suffisamment crédible pour le public visé, à savoir un lectorat de non-spécialistes ? C'est aussi l'avis des collègues pour qui ces erreurs ne nuisent pas à l'efficacité du thriller. Au contraire, le saupoudrage "d'effets FASP » contribue à créer une atmosphère de travail scientifique très lisible pour ceux qui aiment ce type de littérature. Les lecteurs de "techno thrillers" les consomment pour se divertir et non pas comme une leçon scientifique. Mais l'ancrage dans la science est important pour accentuer la peur : le fait qu'il s'agisse d'un scénario possible renforce l'angoisse du lecteur. C'est cet ancrage dans la science qui rend cette fiction si efficace, et les failles au sein de sa représentation ne semblent pas nuire à cette efficacité.

\section{Conclusions}

Les collègues scientifiques ont trouvé que, en matière de substrat professionnel, La proie constitue un "contre-modèle» ou "contre-exemple». La représentation du milieu scientifique est peu crédible mais pourrait servir de point de départ pour une discussion et un travail de la part de l'étudiant. Ce dernier apprendrait à valider lui-même les différents aspects du roman en procédant comme nous l'avons fait. Guidé dans ses recherches, il pourrait corriger les inexactitudes du récit, ce qui l'aiderait dans la préparation de son insertion professionnelle. En plus de ce travail de validation, l'étudiant pourrait s'interroger sur la représentation de la science dans la fiction populaire, notamment sur ce rapport récurrent entre les avancées technologiques et nos peurs les plus fondamentales. Comme l'a écrit Donna Spalding Andréolle dans « How Scientific is Science Fiction? » :

L'étude de tels textes nous donne la possibilité d'examiner la représentation de la science dans la culture populaire, mais aussi de se poser des questions importantes qui en découlent: quel est le rôle de la science dans l'évolution de la société ? Quelles sont les responsabilités des scientifiques dans cette évolution? Les scientifiques devraient-ils se préoccuper de la représentation éthique de leurs découvertes? Et ainsi de suite ${ }^{13}$. (Spalding, 2004, p. 157)

Quant à l'auteur de science-fiction, quelle influence a-t-il sur l'évolution de la société ? Et quelles responsabilités? Un écrivain réagit-il de façon responsable ou éthique quand il se sert de nos peurs pour construire une intrigue ? Sert-il le public quand il mélange à la façon de Michael Crichton science et fiction? Ou bien, les lecteurs sont-ils suffisamment lucides et avertis pour que la fonction de cette littérature demeure ludique? Et si oui, alors pourquoi les médias invitent-ils ces « experts » sur leurs plateaux pour discuter de 
problèmes de fond? Ce ne sont peut-être pas les nanosciences qui sont un "danger " pour l'humanité, mais leur représentation par un auteur de techno thriller, où les priorités fictionnelles, commerciales et politiques prennent le dessus sur les éléments scientifiques.

Enfin, si le manque de crédibilité scientifique dans La proie ne pose pas problème en ce qui concerne la classification du roman en tant que techno-thriller ou science-fiction, il pose problème quant à l'étiquette FASP. Dans les trois cas, des avancées scientifiques et technologiques servent de point de départ dans la création d'un monde à la fois familier et étrange, mais la science-fiction et son sous-genre le techno-thriller offrent une plus grande marge de manœuvre quant à la représentation de la science. En matière de FASP, cette représentation se doit d'être fidèle à la réalité. Il ne s'agit plus de simple « effets de réel », mais d'une convergence du monde professionnel et du thriller. Dans La proie, les éléments qui créent le suspens, à savoir la course contre la montre pour comprendre le fonctionnement de l'essaim de nanoparticules et pour le détruire, et ceux qui réveillent nos peurs, en l'occurrence la perte de contrôle d'une technologie que nous avons créée et l'invasion de notre corps par cette technologie, sont les éléments qui prédominent. Notre enquête permet de conclure que, dans le cas de La proie, Michael Crichton se sert d'éléments scientifiques comme autant de «baromètres de Flaubert » afin de créer un univers professionnel plausible, mais pas tout à fait conforme à la réalité.

\section{Post-scriptum : quelques mots sur la traduction}

21 Comme nous avons travaillé à partir du texte original en anglais et nos collègues à partir de la traduction française, il a fallu comparer les deux versions pour repérer toute différence pouvant induire une interprétation différente, notamment des choix traductionnels qui auraient pu modifier la représentation d'un personnage ou d'un élément scientifique. Nous avons donc procédé à une comparaison détaillée du roman pour finalement constater qu'il n'y avait aucune différence fondamentale. Quelques observations cependant :

- Les premières notes de bas de page dans l'introduction ne paraissent pas dans la traduction française. Il s'agit d'exemples ou de références bibliographiques : un exemple d'incertitude quant aux effets de nos actes, en l'occurrence dans le contexte de la bourse avec une référence à un livre de John Casti, Would Be Worlds (NY, Wiley, 1987, 80+) ; les exemples des deux autres espèces qui possèdent une conscience de soi (les chimpanzés et les orangsoutans) et enfin la référence à l'article scientifique révélant la création involontaire d'un virus mortel par des chercheurs australiens. Ces exemples ont sans doute été jugés superflus, et les références sans réel intérêt pour un public francophone.

- Au niveau des titres des différentes parties, Prey a été traduite par Proies, au pluriel et Home par Famille.

- Le découpage en paragraphes est parfois modifié ainsi que le discours direct et indirect ( Of course not », I said = Je l'en avais assuré. « You're not... » = il m'avait demandé si...).

- Il y a quelques imprécisions dans les choix lexicaux (a chugging = un ronflement ; VC ou Venture capitalists = banquiers, puis capital-risqueurs).

- Quelques ajouts, étoffements, explicitations voire des surtraductions ou «belles infidèles » : le traducteur ajoute l'image d'un ver pour représenter plus clairement les agitations du bébé qui se tortille; il ajoute une chaise dans la chambre de Nicole pour qu'elle puisse la renverser de colère. 
- Il y a quelques petites omissions, quelques faux-sens et quelques modulations sans incidence.

Pour ce qui est du vocabulaire technique ou spécialisé, nos interlocuteurs n'ont rien remarqué de surprenant. La seule question soulevée à plusieurs reprises a concerné le microscope (STM/SEM ou scanning tunneling microscope/scanning electron microscope) et cette erreur ne relevait pas d'un problème de traduction. Nous avons contacté Robert Laffont pour entrer en contact avec le traducteur Patrick Berthon (traducteur des romans de John Grisham et de Robert Silverberg) pour l'interroger sur son travail, mais il est décédé au cours de l'été 2008.

\section{BIBLIOGRAPHIE}

BARTHES, Roland, « L’Effet de réel », Communications,n 11, 1968, p. 84-89.

BENOIT-BROWAEYS, Dorothée, « Nanotechnologies, le vertige de l'infiniment petit », Le monde diplomatique, mars 2008.

BONTOUR, Brigit, « Nanoterreur. La proie de Michael Crichton », disponible sur <http://ecritsvains.com/critique/bontour58.html> [consulté le 31/05/2009].

CHARPY, Jean-Pierre, « FASP médicale et substrat professionnel : le miroir éclaté », ASp, n 57, 2010, 49-65.

CRICHTON, Michael, The Prey, London, HarperCollins, 2002.

CRICHTON, Michael, La proie, Traduit de l'américain par Patrick Berthon, Paris, Robert Laffont, 2003.

CRICHTON, Michael, « Medical Obfuscation: Structure and Function », New England Journal of Medecine, vol. 293, n² 24, 11 décembre 1975.

CRICHTON, Michael, « Host Factors in "Chromophobe" Adenoma of the Anterior Pituitary: A Retrospective Study of 464 Patients ", Metabolism, vol. 30, n 3, mars 1981, p. 248-267.

DREXLER, K. Eric, Engines of Creation. The Coming Era of Nanotechnology, New York, Anchor Press, 1986.

DREXLER, K. Eric, « The Challenge of Nanotechnology », conférence donnée à Seattle, Washington, USA, 17-19 février 1989 à NanoCon (Northwest regional nanotechnology conference) en tant que guest speaker. Disponible sur <http://www.halcyon.com/nanojbl/...>, [consulté le 01/06/2009]. FARMER, J. Doyne et BELIN, Alletta d'A., « Artifical Life: The Coming Evolution » dans Artificial Life II, C. Langton, C. Taylor, J. Farmer et S. Rasmussen (dir.). SFI Studies in the Sciences of Complexity. Proceedings, vol. X, Redwood City, Ca : Addison-Wesley, 1992.

FEYNMAN, Richard, « There's Plenty of Room at the Bottom », conférence donnée devant l'American Physical Society, le 29 décembre 1959 à Caltech, Pasadena, Californie, publiée dans Engineering and Science, février 1960. Disponible sur <http://www.zyvex.com/nanotech/ feynman.html>, [consulté le 01/06/2009]. 
FIEVET, Cyril, «La Proie », critique datée du 25 septembre 2004. Disponible sur <http:// www.nanoblog.com/past/2004/09/la_proie.htm>, [consulté le 31/05/2009].

JoY, Bill, « Why the Future Doesn't Need Us », Wired, avril 2000. Disponible sur <http:// www.wired.com/wired/archive/8.04/joy.html>, [consulté le 31/05/2009].

KIPEN, David, «Crichton Stretches Out Another Nano-Idea », San Francisco Chronicle, 24 novembre 2002. Disponible sur <http://www.sfgate.com/...>, [consulté le 31/05/2009].

LEZARD, Nicolas, « The Admirable Crichton », The Guardian, 14 décembre 2002. Disponible sur < http://www.guardian.co.uk/books/...>, [consulté le 31/05/2009].

мEммот, Carol, «Crichton's "Prey" is High on Tech, Low on Terror », USA Today, 24 novembre 2002. Disponible sur <http://www.usatoday.com/life/books/...>, [consulté le 01/06/2009].

MENGA, David, « Pourquoi les nanotechnologies ? ». Disponible sur <http://

www.internetactu.net/2003/...>, [consulté le 18/05/2009].

MORTON, Oliver, « Dust Devils », Critique de The Prey, The New Yorker, 2 décembre 2002. Disponible sur <http://www.newyorker.com/archive/...>, [consulté le 31/05/2009].

Nanotech Report, juillet 2006. Disponible sur <http://www.qsinano.com/pdf/

ForbesWolfe_NanotechReport_July2006.pdf>, [consulté le 01/06/2009].

NIGEL, critique de The Prey, 5 janvier 2003. Disponible sur <http://www.booklore.co.uk/

PastReviews/...>, [consulté le 31/05/2009].

PETIT, Michel, « La fiction à substrat professionnel : une autre voie d'accès à l'anglais de spécialité », ASp, n²3-26, 1999, p. 57-81.

PETIT, Michel, « Quelques réflexions sur la fiction à substrat professionnel : du général au particulier ", dans PETIT, Michel et Shaeda ISANI, Aspects de la fiction à substrat professionnel, Bordeaux, université Victor Ségalen Bordeaux 2, 2004, p. 3-23.

PHoEnIX, Chris, « Don't Let Crichton's Prey Scare You - The Science Isn't Real », janvier 2003. Disponible sur <http://www.nanotech-now.com/Chris-Phoenix/...>, [consulté le 31/05/2009].

Prey(The),critique anonyme disponible sur<http://contemporarylit.about.com/...>, [consulté le 01/06/2009].

Proie (La), critique anonyme disponible sur <http://www.actualitte.com/dossiers/...>, [consulté le 31/05/2009].

SPALDING ANDRÉOLLE, Donna, « How Scientific is Science Fiction? From "Scientifiction" to Cyberpunk and Beyond", dans PETIT, Michel et SHAEDA Isani, Aspects de la fiction à substrat professionnel, Bordeaux, université Victor Ségalen Bordeaux 2, 2004, p. 153-164.

\section{Sitographie}

<http://www.actu-environnement.com/...> [consulté le 26/04/2010].

<http://www.annuel-idees.fr/NBIC-Les-nouvelles-frontieres-de.html>, [consulté le 26/04/2010].

<http://www.atalayainstitute.org/Alletta\%20Belin.html>, site consacré au travail d'Alletta Belin, coauteur de l'article sur la vie artificielle avec J. Doyne Farmer, [consulté le 18/05/2009].

$<$ http://www.cscs.umich.edu/>,page du Centre pour l'étude des systèmes complexes de l'université de Michigan, qui abrite le groupe Swarm (Swarm development group), « a resource for agent- and individual-based modelers ", [consulté le 18/05/2009].

$<$ http://www.depauw.edu/sfs/>, site de Science Fiction Studies (revue), [consulté le 27/04/2010]. 
<http://www.depauw.edu/sfs/...>, article de Marc ANGENOT et de Dark suvin dans Science Fiction Studies (1979), [consulté le 27/04/2010].

$<$ http://devolab.msu.edu/>, site du Digital evolution lab de Michigan State University. Le laboratoire a été fondé en 1999 par Charles Ofria et Richard Lenski (suite du Caltech Digital Life Lab) et le Experimental Evolution Lab de Lenski. Utilise la plateforme de recherche Avida, [consulté le 18/05/2009].

<http://www.diffusion.ens.fr/index.php?idconf=1275\&res=conf>, conférence d'Irène Langlet (université de Rennes II) à l'ENS rue d'Ulm, le 12 mai 2006 sur « Les états du novum sciencefictionnel : encodages littéraires, décodages lecturaux », [consulté le 27/04/2010].

<http://en.wikipedia.org/wiki/Ray_Kurzweil>, site sur Raymond Kurzweil, inventeur et futuriste, responsable de l'invention de la reconnaissance optique de caractères, de la reconnaissance vocale, des synthétiseurs électroniques et auteur d'ouvrages sur l'intelligence artificielle. Théoricien du transhumanisme et de la singularité technologique, [consulté le 31/05/2009].

<http://www.foresight.org/> Foresight Institute (site d'Eric Drexler), [consulté le 26/04/2010].

$<$ http://informatics.indiana.edu/larryy/al4ai/>, détail du cours « Artificial Life as an Approach to Artificial Intelligence » dispensé à Indiana University (janvier - avril 2009) par Dr Larry Yaeger, un auteur cité par M. Crichton, [consulté le 18/05/2009].

<http://www.innovationlejournal.fr/spip.php?article2291>.

<http://www.internetactu.net/2009/...>

$<$ http://www.laffont.fr/index.asp>, site de l'éditeur consulté pour contact avec traducteur des romans de M. Crichton, Patrick Berthon, mort en août 2008, [consulté le 18/05/2009].

<http://www.michaelcrichton.net/video-charlierose-11-26-02.html>, lien fourni sur le site de l'auteur à l'entretien avec Michael Crichton daté du 26 novembre 2002, [consulté le 01/06/2009].

<http://www.michaelcrichton.net/qa-prey.html>, entretien avec Crichton à propos de La proie, disponible sur le site de l'auteur, [consulté le 01/06/2009].

<http://www.minatec.com/>, site de Minatec, à Grenoble, Pôle d'innovation en micro- et nanotechnologies créé avec le CEA-Leti et Grenoble INP, [consulté le 31/05/2009].

$<$ http://www.nanotechproject.org/>, Project on Emerging nanotechnologies.

<http://www.nsf.gov/crssprgm/nano/...>, [consulté le 26/04/2010].

<http://www.piecesetmaindoeuvre.com/spip.php?page=plan>, site du groupe anti- « nécrotechnologies » de Grenoble qui milite notamment contre Minatec, [consulté le 31/05/2009].

<http://www.santafe.edu/...>, biographie de J. Doyne Farmer, professeur dont les recherches concernent les systèmes complexes, [consulté 18/05/2009].

<http://www.swarm.org/>, site du festival annuel « Swarmfest » qui regroupe tous ceux qui utilisent le logiciel Swarm, [consulté le 18/05/2009].

\section{NOTES}

1. L'idée vient de Jean-Pierre Charpy, «FASP médicale et substrat professionnel : le miroir éclaté », ASp 57, 2010, p. 49-65. Il note comment les «medical thrillers » s'éloignent de plus en 
plus du substrat professionnel médical au profit des éléments empruntés aux «forensic thrillers » et « legal thrillers », d'où la notion « d'hybride ».

2. "Just as war is too important to be left to the generals, science is too important to be left to the scientists ». Citation de Michael Crichton dans un entretien sur La proie trouvé sur le site officiel de l'auteur. <http://www.michaelcrichton.net/qa-prey.html>

3. C'était le cas de Michael Crichton lors de la publication d'État d'urgence (2004). L'auteur a été invité sur les plateaux de télévision américaine en tant qu'expert pour parler du climat (Today Show de NBC et 20/20 d'ABC), ce qui a été dénoncé par la communauté scientifique. Voir le site du Natural Resources Defense Council. «Michael Crichton's State of Fear: They Don't Call It Science Fiction for Nothing ». <http://www.nrdc.org>

4. "Medical writing is a highly skilled, calculated attempt to confuse the reader" "Medical obscurity may serve an intragroup recognition function, rather like a secret fraternal handshake. »Voir Crichton 1975.

5. <http://www.michaelcrichton.net/video-charlierose-11-26-02.html>

6. Si l'article de Bill Joy est né d'une conversation avec Ray Kurzweil, les deux hommes ne partagent pas la même position par rapport aux nanotechnologies. Tandis que Bill Joy nous alerte aux risques liés aux nanotechnologies, Ray Kurtweil est l'un de leurs plus ardents défenseurs. Eric Drexler, dans son livre Engines of Creation, publié en 1986, nous mettait déjà en garde quant aux dangers associés à cette technologie et a fondé le Foresight Institute justement pour les prévenir. 7. Notamment Les nanosciences, tomes 1, 2 et 3 publiés en collaboration avec Marcel Lahmani et Claire Dupas chez Belin, 2006-7. Le tome 2 a gagné le Prix Roberval Enseignement supérieur 2008. 8. NBIC est un sigle qui désigne la convergence des nanosciences, de la biologie, des sciences de l'information et des sciences cognitives (nano-bio-info-cogno). Pour certains, le métissage de ces domaines est très prometteur sur le plan scientifique et industriel et annonce une évolution qui bouleversera l'avenir de l'humanité ; d'autres craignent les risques de ce métissage et cherchent à établir des contrôles internationaux. Voir <http://www.nsf.gov/crssprgm/nano/ global_govt_online_jnr.pdf>

9. Voir, par exemple le site : <http://www.internetactu.net/2009/...>

10. <http://www.innovationlejournal.fr/spip.php?article2291>

11. <http://www.actu-environnement.com/...>

12. L'expression « effet FASP " s'inspire de «l'effet de réel » de Roland Barthes qui relève des contraintes $\mathrm{du}$ réalisme, dont l'exemple-type est le baromètre de Flaubert. Le baromètre suspendu au-dessus du piano dans le conte de Flaubert analysé par Barthes n'a aucune fonction dans le récit sauf comme élément de décor. Voir Barthes, Roland, "L'Effet de réel", Communications, 11, 1968, p. 84-89.

13. "The study of such texts gives the opportunity not only to examine how science is represented in the popular culture, but also to approach serious social issues emanating from scientific progress: what is science's role in the shaping of society? What are the scientist's responsibilities in this process? Should the scientist be concerned with the ethical representation of his/her discoveries? And so forth.» 


\section{RÉSUMÉS}

La FASP constitue un genre de fiction spécifique de par son contenu "spécialisé » ou "professionnel», contenu qui côtoie des éléments de fiction au sein d'une logique de thriller. Mais comment évaluer l'authenticité de ce contenu, surtout dans un objectif pédagogique, si l'on est non-spécialiste? Une issue évidente est le recours aux spécialistes eux-mêmes. Nous avons décidé de mener une enquête auprès de collègues scientifiques travaillant dans la nanotechnologie, la biologie et l'intelligence artificielle, les domaines décrits dans La proie (Prey, 2002) de l'auteur américain Michael Crichton. Nous nous sommes penchés sur la crédibilité de l'intrigue, sur la représentation de la technologie et de la recherche, des lieux, des personnages et de leur comportement et, enfin, sur la langue spécialisée. Nous avons aussi voulu voir dans quelle mesure Crichton réussit à élaborer un substrat qui se situe en dehors de ses propres compétences scientifiques afin de tester la notion de "FASP hybride ${ }^{1}$ ». Enfin, puisque nos collègues ont préféré lire le texte en français, nous avons été amenée à travailler sur la traduction du texte américain.

Professional-based fiction is a type of thriller in which "specialized" content exists alongside fictional elements. But how can we assess the authenticity of this content, especially in a pedagogical aim, if we ourselves are non-specialists? One answer is to call upon professionals. We decided to survey scientists working in nanotechnology, biology and artificial intelligence, domains described in the Prey (2002) by American author Michael Crichton. We analyzed the legitimacy of the plot, the representation of technology and research, the places, characters and their behavior and, finally, the specialized language. We also wanted to see how well Crichton succeeds in portraying fields outside of his own scientific training in order to test the notion of "hybrid" professional-based fiction (Charpy, 2010). And, since our colleagues preferred to read the novel in French, we had to consider the translation of the text.

\section{INDEX}

Mots-clés : FASP, Michael Crichton, nanotechnologies, techno-thriller

Keywords : Professional-based fiction, Michael Crichton, nanotechnologies, techno-thriller 